\title{
The imitation game: Pulmonary artery sarcoma masquerading as an acute pulmonary embolism
}

Stevan S. Pupovac, MD, ${ }^{a}$ Jonathan M. Hemli, MD, ${ }^{a}$ I. Claire Sarmiento, BSN, ${ }^{b}$ Richard S. Lazzaro, MD, S. Jacob Scheinerman, MD, ${ }^{b}$ and Derek R. Brinster, MD, ${ }^{b}$ Manhasset and New York, NY

\footnotetext{
From the ${ }^{\mathrm{a}}$ Department of Cardiothoracic Surgery, North Shore University Hospital/Northwell Health, Manhasset; and ${ }^{\mathrm{b}}$ Department of Cardiovascular \& Thoracic Surgery, Lenox Hill Hospital/Northwell Health, New York, NY

Disclosures: Dr Brinster is a consultant for Terumo Aortic, Cook Medical, and W. L. Gore \& Associates. All other authors reported no conflicts of interest.

The Journal policy requires editors and reviewers to disclose conflicts of interest and to decline handling or reviewing manuscripts for which they may have a conflict of interest. The editors and reviewers of this article have no conflicts of interest.

Read at the 101st Annual Meeting of The American Association for Thoracic Surgery: A Virtual Learning Experience, April 30-May 2, 2021.

Received for publication June 2, 2021; accepted for publication Aug 27, 2021; available ahead of print Sept 6, 2021.

Address for reprints: Stevan S. Pupovac, MD, Department of Cardiothoracic Surgery, North Shore University Hospital, 300 Community Dr, Manhasset, NY 11030 (E-mail: spupovac@northwell.edu)

JTCVS Techniques 2021;10:317-9

2666-2507

Copyright (C) 2021 The Author(s). Published by Elsevier Inc. on behalf of The American Association for Thoracic Surgery. This is an open access article under the CC BY-NC-ND license (http://creativecommons.org/licenses/bync-nd/4.0/).

https://doi.org/10.1016/j.xjtc.2021.08.039
}

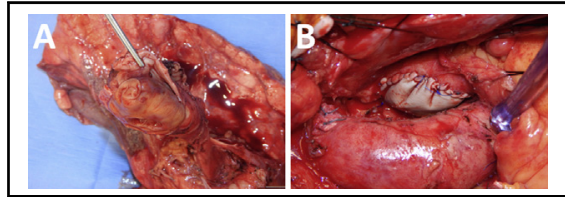

Right pulmonary artery sarcoma. Before resection (A) and following reconstruction (B).

CENTRAL MESSAGE

Pulmonary artery sarcoma (PAS)

typically carries a poor prognosis.

Surgical resection and adjuvant

multimodality therapy offer the

best chance at long-term sur-

vival, but diagnosis can be

difficult.

See Commentary on page 320.
Video clip is available online.

Primary sarcoma of the pulmonary artery is rare $(0.001 \%$ $0.03 \%$ of the population) and has been typically associated with a dismal prognosis; after surgical resection and adjuvant therapy, median and 5-year survival have been reported as 20 months and $22 \%$, respectively. ${ }^{1}$ Treatment often tends to be palliative and symptom-directed, aiming to restore pulmonary blood flow to address any ventilation-perfusion mismatch and to reduce right-sided heart strain. ${ }^{1,2}$ Herein, we discuss an interesting case of a pulmonary artery sarcoma (PAS) that was initially mistaken for a submassive pulmonary embolism but which was nevertheless managed successfully with better-than-expected oncologic outcomes (as seen in Video 1).

\section{CASE PRESENTATION}

A 44-year-old otherwise-healthy male was emergently taken to the operating room with a working diagnosis of acute submassive pulmonary thromboembolism, with radiologic evidence of a large occlusive mass in the right pulmonary artery (Figure 1), complicated by borderline hemodynamic stability, significant right ventricular strain on echocardiography, and elevated biochemical markers suggestive of myocardial injury.
Catheter-directed thrombolysis was considered; however, our concern with this strategy in a younger patient was the potential to fragment the large proximal occlusive mass and place the patient at risk for chronic thromboembolic pulmonary hypertension. However, we have had quite

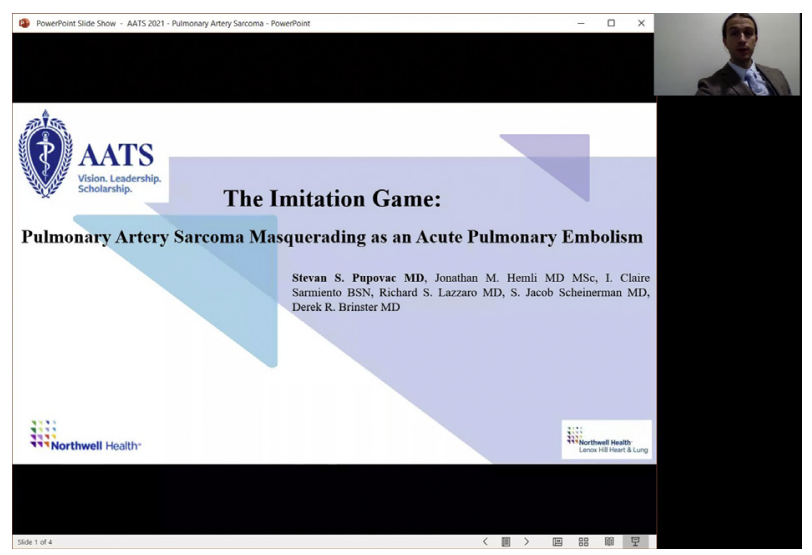

VIDEO 1. Author's case presentation from the American Association for Thoracic Surgery 101st Annual Meeting Resident Case Report competition. Video available at: https://www.jtcvs.org/article/S2666-2507(21) 00599-X/fulltext. 

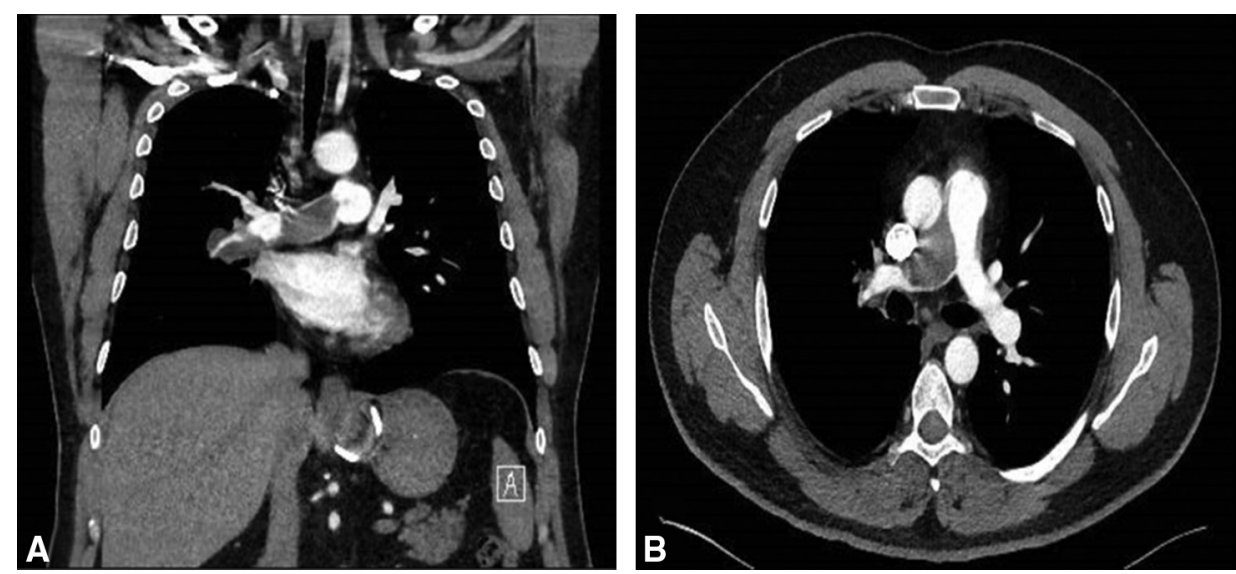

FIGURE 1. Computed tomography of the chest in coronal (A) and axial (B) planes, demonstrating an occlusive mass in the right pulmonary artery.

positive results using the FlowTriever system (Inari Medical, Irvine, Calif), as the catheters of this device are more flexible and seem more easily positioned up into the pulmonary arteries, with less trauma and torque, as compared with the AngioVac (AngioDynamics, Latham, NY). Unfortunately, we did not have this device available to us at the time that our patient presented. Bearing in mind that our working diagnosis was venous thromboembolism, and that our patient was young and otherwise healthy, we preferred to surgically remove the entire clot burden and thereby minimize any potential chances of chronic thromboembolic pulmonary hypertension in the longer term.

Standard techniques of cardiopulmonary bypass were employed, without the need for circulatory arrest. Initial dissection of the aortopulmonary window revealed a fibrotic density, distorting normal tissue planes, which was concerning for malignancy. Frozen section pathologic examination of an operative biopsy was suggestive of a sarcomatoid neoplasm. When the main pulmonary trunk was opened, however, it was clear that the luminal mass was a large tumor, extending from the pulmonary bifurcation all the way to the right hilum; consequently, a radical right pneumonectomy was undertaken, with en bloc intrapericardial resection of the right pulmonary artery in its entirety. The right ventricular outflow tract and main pulmonary artery were reconstructed with xenograft pericardium, preserving the native pulmonic valve (Figure 2). Histopathology was consistent with a high-grade intimal sarcoma of the pulmonary artery; the proximal resection margin was negative for tumor involvement. After an uncomplicated postoperative recovery, the patient completed a course of doxorubicin and imatinib.

Twenty-eight months postresection, while the patient was being maintained on imatinib therapy, surveillance imaging demonstrated a new, partially obstructing mass in the right ventricular outflow tract, adjacent to the pulmonic valve, as well as a solitary lesion in the left iliac crest, consistent with recurrent/metastatic disease. The patient underwent reoperative sternotomy and radical excision of the entire pulmonic root, incorporating pulmonic valve replacement and extensive reconstruction of the right ventricular outflow tract. Histopathologic examination confirmed recurrent high-grade intimal sarcoma; surgical resection margins were again negative for tumor. Two months thereafter, he received stereotactic radiation to the iliac lesion.

Currently, at 36 months since his primary operation, the patient remains on combination chemo- and immunotherapy, namely, trastuzumab (a monoclonal antibody used in HER2
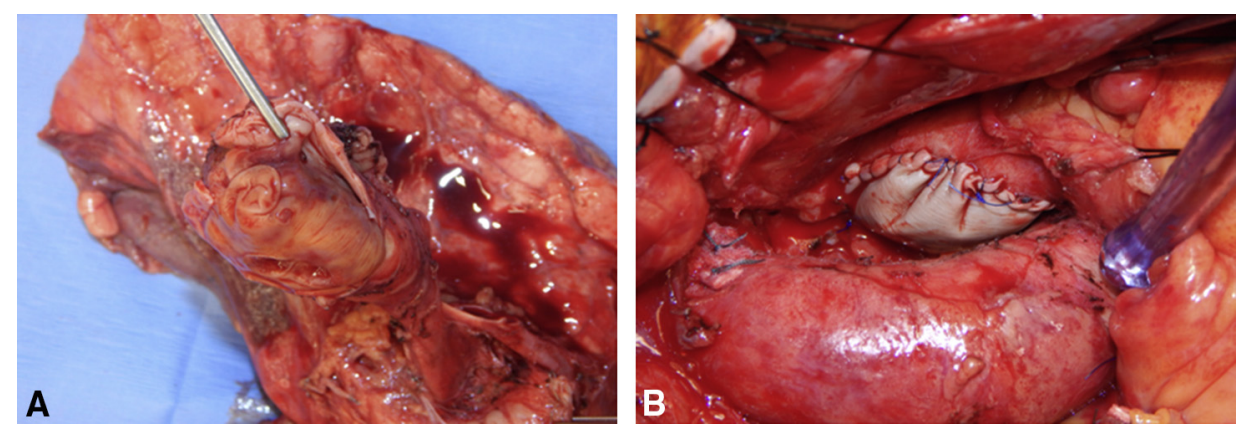

FIGURE 2. A, Radical right pneumonectomy with en bloc resection of the right pulmonary artery; the vessel is completely occluded by tumor. B, Reconstruction of the main pulmonary artery and right ventricular outflow tract with xenograft pericardium. 
positive tumors), gemcitabine (an anti-metabolite that inhibits DNA synthesis), osimertinib (a kinase inhibitor of the epidermal growth factor receptor), and oxaliplatin (a platinum-based alkylating agent that disrupts DNA replication and transcription) and has no clinical or radiologic evidence of residual disease. Individual patient consent was obtained for this report.

\section{DISCUSSION}

The first documented case of PAS was described by Mandelstamm in $1923 .^{3}$ Given the rarity of the condition, coupled with its similar clinical and radiographic appearance to venous thromboembolic disease, preoperative diagnosis may be challenging. ${ }^{2}$

PAS is typically diagnosed at an advanced stage of disease, with pulmonary metastases present in greater than $50 \%$ of patients and distant metastases evident in $16 \%$, at the time of diagnosis. ${ }^{4}$ Consequently, PAS is usually not amenable to curative resection, and management is primarily geared toward palliation; the median survival of untreated patients has been reported as short as 1.5 months. ${ }^{5}$

Aggressive surgical resection remains the only option for potential cure, with isolated reports of survival more than 70 months after combined surgical resection and adjuvant multimodality therapy. ${ }^{2}$ In their review of the literature, Blackmon and colleagues ${ }^{2}$ reported a median survival of $36.5 \pm 20.2$ months for patients undergoing an attempt at curative resection versus $11 \pm 3$ months for those who had incomplete tumor removal. Median survival was $24.7 \pm 8.5$ months for those that received multimodality treatment versus $8.0 \pm 1.7$ months for those who had single-modality therapy only. If complete resection is unachievable, management is focused on limiting the development of pulmonary hypertension, right ventricular dysfunction, and progressive right heart failure and may include a combination of palliative pulmonary artery stenting, tumor debulking, and/or thromboendarterectomy.,

Extended tumor-free survival is rare and the treatment for recurrence is ill-defined. In our patient, surveillance imaging prompted further surgical resection, followed by targeted radiation and systemic chemo/immunotherapy. Given that our patient remains alive at 36 months following his initial diagnosis, without evidence of residual disease, we affirm that aggressive surgical resection, coupled with multimodality adjuvant therapy, can be effective in managing PAS.

\section{CONCLUSIONS}

PAS portends a poor prognosis. Nevertheless, improved longer-term survival is possible after prompt diagnosis, aggressive surgical resection (including repeat resection for recurrent/metastatic disease), and adjuvant multimodality therapy.

\section{References}

1. Mussot S, Ghigna M, Mercier O, Fabre D, Fadel E, Le Cesne A, et al. Retrospective institutional study of 31 patients treated for pulmonary artery sarcoma. Eur $J$ Cardiothorac Surg. 2013;43:787-93.

2. Blackmon SH, Rice DC, Correa AM, Mehran R, Putnam JB, Smythe WR, et al Management of primary pulmonary artery sarcomas. Ann Thorac Surg. 2009; 87:977-84.

3. Mandelstamm M. Uber primare neubildungen des herzens. Virchows Arch. 1923; 245:43-54

4. Seol SH, Jeong N, Kim I, Kim J. Pulmonary artery sarcoma-multimodality imaging. J Clin Imaging Sci. 2016;6:45

5. Gan HL, Zhang JQ, Zhou QW, Xiao W, Gao YM, Liu S, et al. Surgical treatment of pulmonary artery sarcoma. J Thorac Cardiovasc Surg. 2011;142:1469-72. 